\title{
New Insights into the Structure of Nanoporous Carbons from NMR, Raman, and Pair Distribution Function Analysis
}

\author{
Alexander C. Forse ${ }^{\text {a }}$, Céline Merlet ${ }^{a}$, Phoebe K. Allan ${ }^{\text {a,c }}$, Elizabeth K. Humphreys ${ }^{a}$, John M. Griffin ${ }^{\text {a }}$, Mesut

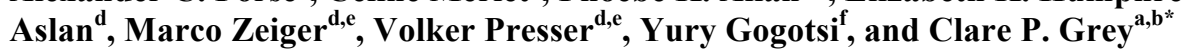 \\ ${ }^{a}$ Department of Chemistry, University of Cambridge, Lensfield Road, Cambridge, CB2 1EW, UK. \\ ${ }^{b}$ Department of Chemistry, Stony Brook University, Stony Brook, NY 11794, USA. \\ ${ }^{c}$ Gonville and Caius College, Trinity Street, Cambridge, CB2 1TA, UK. \\ ${ }^{d} I N M$ - Leibniz-Institute for New Materials, 66123 Saarbrücken, Germany. \\ ${ }^{e}$ Department of Materials Science and Engineering, Saarland University, 66123 Saarbrücken, Germany. \\ ${ }^{f}$ Department of Materials Science and Engineering and A.J. Drexel Nanomaterials Institute, Drexel University, Philadelphia, PA \\ 19104, USA. \\ *Corresponding author. Email: cpg27@cam.ac.uk
}

\section{Abstract}

The structural characterisation of nanoporous carbons is a challenging task as they generally lack long-range order and can exhibit diverse local structures. Such characterisation represents an important step towards understanding and improving the properties and functionality of porous carbons, yet few experimental techniques have been developed for this purpose. Here we demonstrate the application of nuclear magnetic resonance (NMR) spectroscopy and pair distribution function (PDF) analysis as new tools to probe the local structures of porous carbons, alongside more conventional Raman spectroscopy. Together, the PDFs and the Raman spectra allow the local chemical bonding to be probed, with the bonding becoming more ordered for carbide-derived carbons (CDCs) synthesised at higher temperatures. The ring currents induced in the NMR experiment (and thus the observed NMR chemical shifts for adsorbed species) are strongly dependent on the size of the aromatic carbon domains. We exploit this property and use computer simulations to show that the carbon domain size increases with the temperature used in the carbon synthesis. The techniques developed here are applicable to a wide range of porous carbons, and offer new insights into the structures of CDCs (conventional and vacuum-annealed) and coconut shell-derived activated carbons.

\section{Introduction}

Nanoporous carbons are an important class of materials used in a range of applications including capacitive energy storage, gas storage, water treatment, and catalysis. ${ }^{1-3}$ In each case, the nanoporosity and high specific surface areas (typically $>1500 \mathrm{~m}^{2} \mathrm{~g}^{-1}$ ), achieved by activating carbonaceous precursors, are exploited to store molecules or ions. In principle, carbon structures can be engineered for a given application, though characterisation of the highly disordered structures poses a significant challenge. The challenges in determining local- and long-range structure make it 
extremely difficult to establish structure-function correlations beyond those simply derived from surface area and pore-size distributions.

The structures of carbon materials ${ }^{4,5}$ have been actively researched since the pioneering X-ray diffraction studies of Franklin. ${ }^{6,7}$ She distinguished between graphitizing and non-graphitizing carbons, the former transforming into graphite upon heating to high temperature, and the latter showing no such transformation at temperatures as high as $3000{ }^{\circ} \mathrm{C}{ }^{7}$ For nanoporous carbons, analysis of the broad Bragg peaks is generally of limited use due to the long range disordered structures of these materials. However, inclusion of the diffuse scattering in the analysis allows the extraction of a pair distribution function (PDF), which is a weighted histogram of atom-to-atom distances showing the likelihood of finding an atom-pair separated by a certain distance. ${ }^{8}$ PDF studies show that porous carbons often exhibit a high degree of local ordering, with a propensity for hexagonal carbon rings in which the carbon atoms are $\mathrm{sp}^{2}$ hybridised. ${ }^{9-11}$ Correlations in the PDFs typically extend over tens of Angstroms, suggesting that there is local order on this length scale. ${ }^{10}$ Transmission electron microscopy (TEM) images corroborate these concepts and generally show curved carbon sheets arranged in a disordered fashion. ${ }^{9,12-14}$ The sheet curvature is thought to arise from the presence of non-hexagonal carbon rings, observed in experimental ${ }^{13}$ and modelling studies. $^{15-17}$

Nuclear magnetic resonance (NMR) spectroscopy of adsorbate molecules is emerging as an advanced method to characterise the structure of carbon nanomaterials, ${ }^{18-24}$ as well as to characterise gas storage $^{18,25,26}$ and energy storage $\mathrm{e}^{27-31}$ systems in situ. In NMR spectroscopy, molecules or ions adsorbed inside carbon nanopores give rise to a spectral feature that is distinct from that of nonadsorbed ones. This arises as the delocalized carbon $\pi$ electrons circulate in the presence of an applied magnetic field, inducing a local magnetic field that shields nearby nuclei (referred to as a ring current effect). The chemical shifts observed for different adsorbed species are generally very similar for a given carbon, ${ }^{23,32-34}$ suggesting that the ring current shift is nucleus-independent to a first approximation. This has motivated the use of nucleus-independent chemical shift (NICS) calculations to rationalise the chemical shifts observed for adsorbates. ${ }^{20,22,23,35,36}$ When different carbon structures are studied experimentally, the chemical shifts observed for a given adsorbed molecule can vary dramatically. ${ }^{19,20,22,32}$ Our recent NICS calculations on model carbon fragments, in combination with lattice-simulations, suggested that the carbon pore size and the size of the hexagonally bonded carbon fragments in which ring currents are present can each have significant effects on the shifts observed for adsorbed species. ${ }^{20,24}$

Today, nanoporous carbons are most commonly characterised by gas sorption experiments, ${ }^{37}$ which allow a determination of the carbon pore size distribution, and Raman spectroscopy, which provides 
a measure of disorder in the carbon bonding network. ${ }^{38-40}$ Based on the large Raman cross-section of $\mathrm{sp}^{2}$-hybridized carbon, structural analysis of small amounts of material is possible. Incompletely graphitized carbons show two prominent spectral features: the G-mode between 1580 and $1590 \mathrm{~cm}^{-1}$ indicative of $\mathrm{sp}^{2}$-hybridized carbon (bond stretching of $\mathrm{sp}^{2}$-hybridized carbon atoms in rings and chains coming from the zone centre $E_{2 g}$ mode) and the D-mode between 1330 and $1342 \mathrm{~cm}^{-1}$ which represents the breathing mode of the six-fold carbon rings, but is only Raman active in the presence of defects, such as edges or vacancies. ${ }^{39}$ While the $\mathrm{D} / \mathrm{G}$ peak area ratio can be used as a quantitative measure for the crystallite size in nano-crystalline graphites, ${ }^{41,42}$ for disordered porous carbons, the D- and G-band widths are commonly used as a measure of disorder in the carbon-carbon bonding network. $^{12,43,44}$

Given the complexity and variety of nanoporous carbon structures, there is considerable scope for the development of characterisation tools that can provide more accurate and complete structural information. Here, we investigate the structures of a series of titanium carbide-derived carbons (TiCCDCs) with a range of methods to compare and contrast the information that can be obtained. These materials were chosen for study because it is possible to control the porosity and degree of ordering by varying the synthesis temperature, ${ }^{12}$ and also by introducing additional vacuum annealing steps. ${ }^{14}$ Pair distribution function analysis offers a powerful probe of local chemical structure, providing information which is complementary to that obtained from Raman analysis. These experiments show that the chemical bonding of TiC-CDCs becomes more uniform for materials synthesised at higher temperatures. The chemical shifts observed for adsorbates in NMR are shown to be particularly sensitive to the sizes of the carbon domains over which ring currents are present, and therefore offer a further probe of the local carbon structure. With the NMR approach, variations of the carbon pore size must be taken into account when interpreting the data. We demonstrate a convenient way to do this using a lattice simulation method that allows the size of the carbon domains to be estimated. The carbon domains in which ring currents are present are shown to increase in size with the maximum temperature used in the carbon synthesis. The application of the approach to commercial activated carbons is then demonstrated. 


\section{Results and Discussion}

\section{Probing the structure of TiC-CDCs}
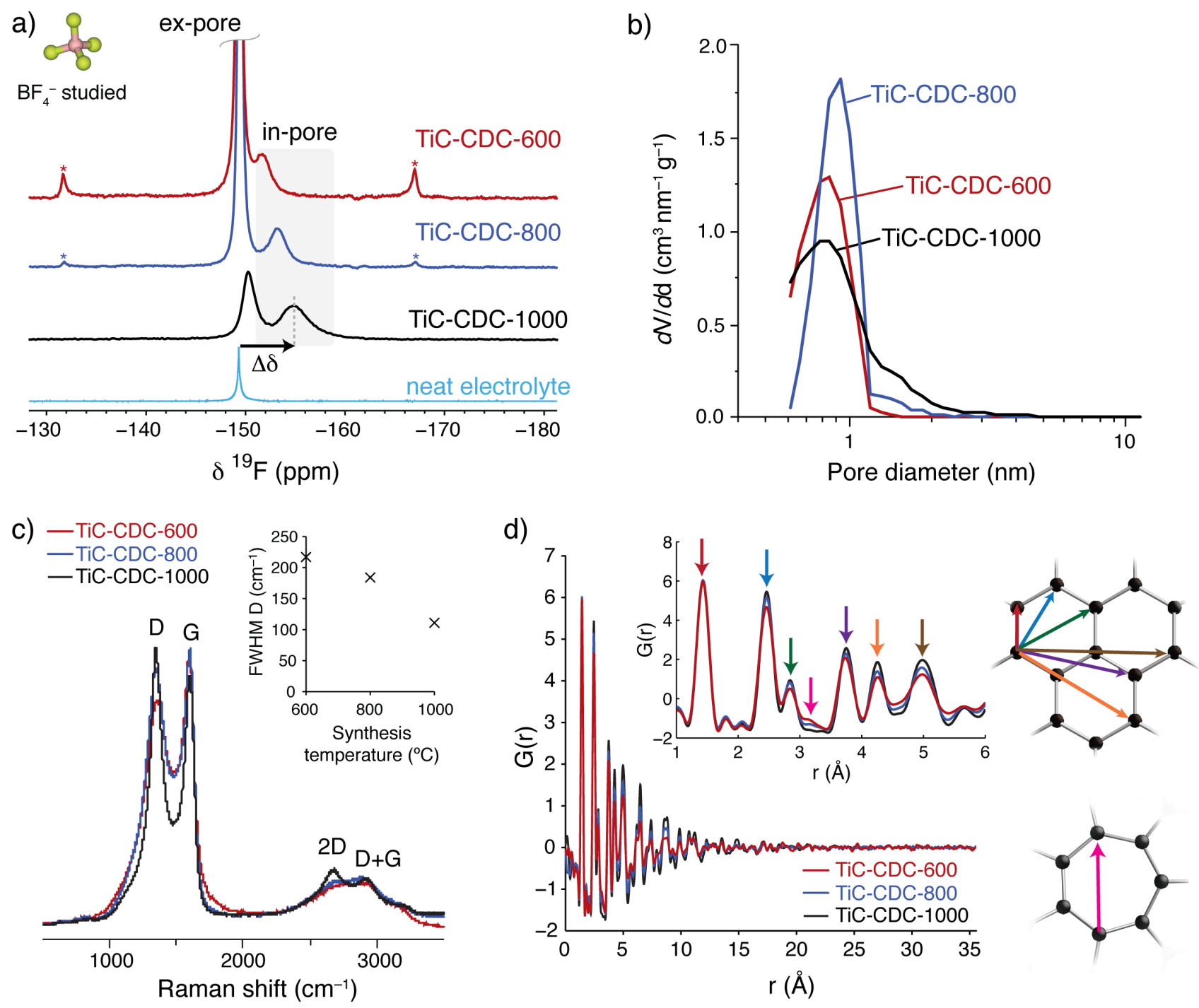

Figure 1 Characterisation of TiC-CDC samples. a) ${ }^{19} \mathrm{~F}$ MAS NMR $(7.1 \mathrm{~T})$ spectra of TiC-CDCs soaked with $\mathrm{NEt}_{4} \mathrm{BF}_{4} / \mathrm{dACN}(1.5 \mathrm{M})$ electrolyte. An example $\Delta \delta$ value is shown for TiC-CDC-1000. The MAS rate was $5 \mathrm{kHz}$, and spinning sidebands are marked by asterisks. The spectrum of neat electrolyte is shown for comparison. b) Pore size distributions measured by $\mathrm{N}_{2}$ gas sorption (see Supporting Information for the sorption isotherms). c) Raman spectra, with the deconvoluted full-width at half-maximum intensity (FWHM) values for the D-band shown in the inset. Full details of the fitted parameters are given in the Supporting Information. d) X-ray PDFs. The inset shows the region between 1 and $6 \AA$ with the assignments to various C-C correlations indicated.

A range of experiments were carried out to characterize the structures of a series of TiC-CDCs, prepared by etching of titanium carbide in chlorine at different temperatures (a sample synthesised at $\mathrm{X}{ }^{\circ} \mathrm{C}$ is referred to as TiC-CDC-X, e.g., TiC-CDC-600). ${ }^{19} \mathrm{~F}$ magic angle spinning (MAS) NMR spectra of TiC-CDCs soaked with tetraethylammonium tetrafluoroborate $\left(\mathrm{NEt}_{4} \mathrm{BF}_{4}\right)$ in deuterated acetonitrile $\left(\mathrm{D}_{3} \mathrm{CCN}\right.$, referred to as $\mathrm{dACN}$ here) $(1.5 \mathrm{M})$ are shown in Figure 1a. In each case, a 
resonance arising from ions nearby carbon surfaces inside carbon nanopores (referred to as "in-pore") is observed, as well as an "ex-pore" resonance arising from ions in large spaces between the carbon particles (see introduction). Here, we define the $\Delta \delta$ value as the chemical shift difference between the in-pore and the neat electrolyte resonances (see Figure 1a). Measured $\Delta \delta$ values are $-2.4,-3.9$ and $5.5 \mathrm{ppm}$ for TiC-CDC-600, -800 and -1000 , respectively, the magnitude of the $\Delta \delta$ value giving a measure of the strength of the ring currents for each carbon. Our choice to report ${ }^{19} \mathrm{~F} \Delta \delta$ values for $\mathrm{BF}_{4}^{-}$is somewhat arbitrary, and values from ${ }^{1} \mathrm{H} \mathrm{NMR}$ spectra of the $\mathrm{NEt}_{4}{ }^{+}$cations reveal similar results, with the $\Delta \delta$ values being nucleus-independent to a first approximation (see Supporting Information). We note that the in-pore line widths also show changes with synthesis temperature, which likely arise from a combination of changes of in-pore ionic diffusion rates, and differences in the profile of adsorption sites (with different distributions of ring current shifts) between the carbons. $^{45}$

Here, we focus our discussion on the $\Delta \delta$ values, which offer information about the carbon structures. Our previous NICS calculations for a range of model carbon fragments showed that carbons with smaller pores give rise to $\Delta \delta$ values of greater magnitude (as the ring current effects from each pore wall are additive) while there is also an effect from carbon ordering, whereby carbons with larger domain sizes (domains of hexagonally bonded carbon in which ring currents are established) also give rise to $\Delta \delta$ values of greater magnitude. ${ }^{20,24}$ In the spectra presented here, the $\Delta \delta$ values increase in magnitude as the CDC synthesis temperature is increased, as in previous work. ${ }^{19,20}$ This increase occurs despite small increases in the carbon pore size, with average pore size values of 8.2, 9.1, and $9.3 \AA$ measured by $\mathrm{N}_{2}$ sorption for TiC-CDC-600, -800, and -1000, respectively (Figure 1b). Instead, this suggests that increases in the sizes of the carbon domains over which the ring currents act dominate the variation of the $\Delta \delta$ values.

Raman spectra of these carbons (Figure 1c) show D- and G-bands typical for disordered porous carbons. As observed previously, ${ }^{12,43,44}$ a decrease of the D-band (a feature associated with defects or disorder in the carbon sheets ${ }^{1,39}$ ) full-width at half-maximum intensity (FWHM) is observed as the chlorine-treatment temperature is increased. This indicates that the carbon-carbon bonding becomes more uniform and less defective as the synthesis temperature is increased.

X-ray PDFs of the carbons are consistent with hexagonally bonded $\mathrm{sp}^{2}$-hybridised carbon (Figure 1d), with correlations extending to distances of approximately $20 \AA$. The loss of the correlations at large distances arises from disorder in the carbon sheets, with a combination of sheet-curvature and termination of the carbon sheets by either hydrogen atoms or functional groups likely responsible. The correlations extend to slightly larger distances for TiC-CDCs produced at higher temperatures, suggesting that the carbon sheets are larger and/or less curved. The FWHMs for the first three peaks 
in the PDFs also show decreases with synthesis temperature, indicating increased order on a very local scale, consistent with the Raman spectra (see Supporting information). The correlations in the PDFs are well modelled by simulations on a terminated graphite crystal, with a large thermal parameter in the c-lattice direction to account for interlayer disorder (see Supporting information). This supports the theory that the carbons consist primarily of $\mathrm{sp}^{2}$-hybrised carbon sheets with hexagonal bonding, and also confirms the model proposed by Petkov et al., ${ }^{10}$ that the correlations in the PDFs are dominated by in-plane carbon-carbon correlations, and that the different sheets (pore walls) are not strongly correlated with each other. Interestingly, small correlations are observed at $\sim 3.2 \AA$ in the PDFs of all three carbons, which are not accounted for in the simulations based on hexagonally bonded carbon. In order to confirm that these correlations were not the result of truncation artefacts, the data were Fourier transformed over a number of Q-ranges and the peak position was unchanged. The observation of this correlation is consistent with the presence of sevenmembered carbon rings (see Supporting Information for more details). These are most apparent for samples synthesised at lower temperatures, and are expected to give rise to a higher degree of sheet curvature for these samples. ${ }^{5}$

Overall, our findings suggest that local carbon-carbon bonding becomes more ordered as the chlorine treatment temperature is increased (Raman and PDF), while the NMR experiments point towards larger carbon domains in which ring currents are present. This latter finding is consistent with previous diamagnetic susceptibility measurements ${ }^{46}$ and quenched molecular dynamics structure simulations. ${ }^{47}$ It also helps to explain the improved conductivity of CDC materials synthesised at higher temperatures, ${ }^{48}$ with the results also mirroring those recently obtained in a study of activated carbon fibres. ${ }^{49}$

\section{The effects of high temperature annealing}

To further investigate the utility of the different characterisation methods, we studied TiC-CDCs which, after synthesis, were vacuum-annealed for one hour at $1400{ }^{\circ} \mathrm{C}$ (samples are then referred to as TiC-CDC-X-VAC; e.g., TiC-CDC-600-VAC). Such high temperature vacuum annealing is thought to increase the size of the hexagonally bonded carbon domains, remove oxygen containing functional groups, and in some cases cause stacking of the defective carbon sheets. ${ }^{14,44}$ Vacuum annealing at this temperature does not lead to a significant loss in carbon porosity and only causes small changes in the carbon pore size distributions, with the resulting porosity largely dependent on the synthesis temperature used. 
a)

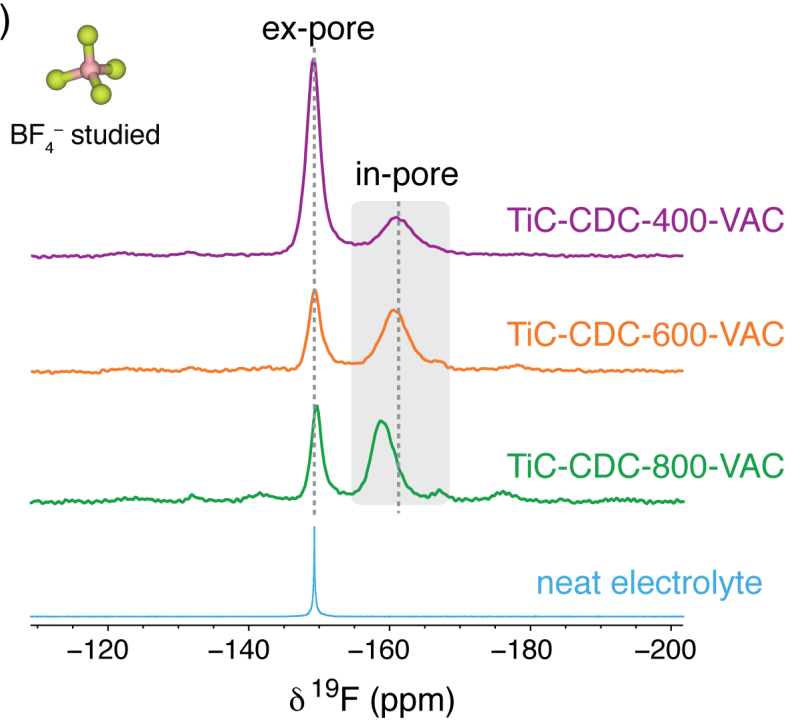

c)

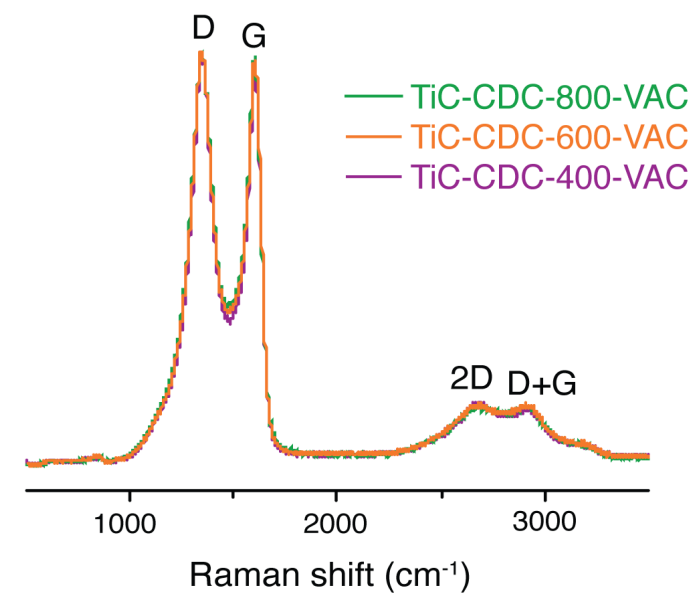

b)

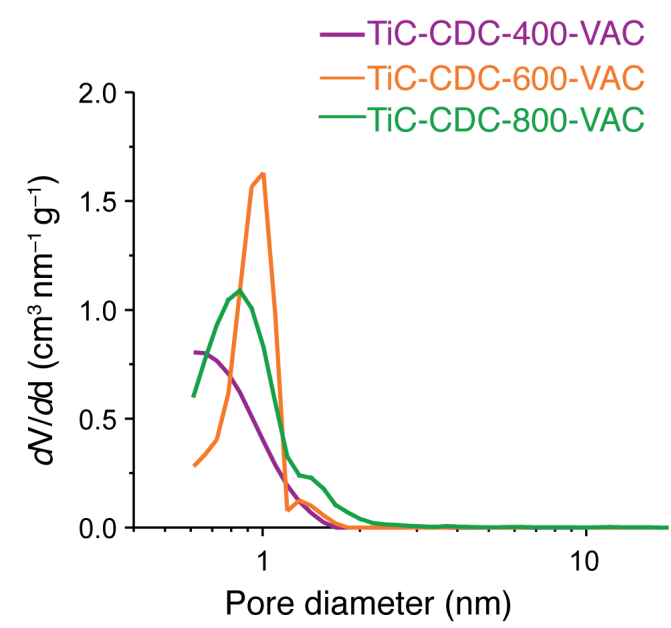

d)

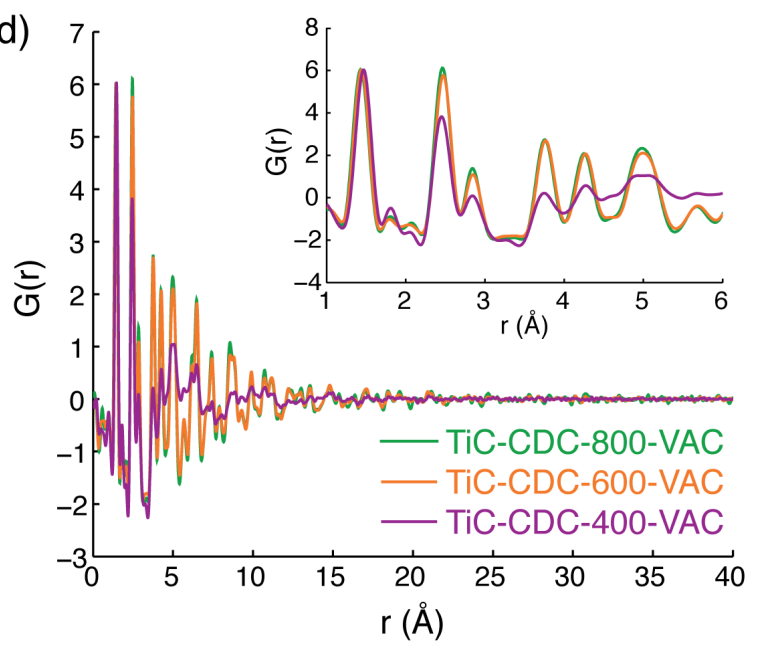

Figure 2 Characterisation of carbide-derived carbon samples which were vacuum-annealed at a common temperature of $1400{ }^{\circ} \mathrm{C}$. a) ${ }^{19} \mathrm{~F}$ MAS NMR (7.1 T) spectra of TiC-CDCs soaked with $\mathrm{NEt}_{4} \mathrm{BF}_{4} / \mathrm{dACN}(1.5 \mathrm{M})$ electrolyte. b) Pore size distributions measured by $\mathrm{N}_{2}$ gas sorption. c) Raman spectra. d) X-ray PDFs.

${ }^{19}$ F MAS NMR spectra of electrolyte-soaked TiC-CDC-VAC samples are shown in Figure 2a, again with a variation of $\Delta \delta$ observed with the sample chlorine-treatment temperature. In contrast to the effect for conventional TiC-CDCs above, here the ${ }^{19} \mathrm{~F} \Delta \delta$ values are (i) much larger in magnitude, and (ii) show decreases in magnitude from -11.6 , to -11.1 , to $-9.7 \mathrm{ppm}$, for TiC-CDC-400-VAC, $600-\mathrm{VAC}$, and 800-VAC, respectively, with very similar behaviour observed in ${ }^{1} \mathrm{H}$ NMR spectra of the $\mathrm{NEt}_{4}$ cations (see Supporting Information). This indicates that (i) the aromatic domains are much larger here, and (ii) the variation of carbon pore size (Figure 2b) may be the dominant source of the observed variation of the $\Delta \delta$ values within the TiC-CDC-VAC series.

The Raman spectra are almost identical for the three carbons (Figure 2c), suggesting that the carboncarbon bonding is very similar for these materials that were vacuum annealed at a common temperature of $1400{ }^{\circ} \mathrm{C}$. X-ray PDFs (Figure 2d) of TiC-CDC-800-VAC and TiC-CDC-600-VAC are 
also very similar, however, the PDF of TiC-CDC-400-VAC is very different, with a more rapid decay of the C-C correlations. Small shifts of the first few correlations are also observed for this carbon, with the first three correlations at $1.46,2.44$, and $2.86 \AA$, compared to $1.43,2.46$, and $2.86 \AA$ for the rest of the carbons studied in this work. The marked differences between the PDFs and the Raman spectra suggest that while the bond vibrations in these carbons are very similar, there are some significant differences in the local chemical structure. These differences may arise from a high degree of curvature in the carbon sheets of the TiC-CDC-400-VAC material, which was chlorine treated at a low temperature of $400{ }^{\circ} \mathrm{C}$. That said, none of the vacuum annealed carbons show clear correlations for 7-membered carbon rings at $\sim 3.2 \AA$, as were observed for normal TiC-CDCs. Importantly, these findings show that Raman spectroscopy alone is insufficient to characterize the structures of carbon materials. We note that the X-ray powder (Bragg) diffraction patterns of these carbons (and the non-annealed carbons) are all very similar, suggesting that there are no significant differences arising from any crystallinity in these samples (see Supporting Information).

From the Raman spectra and PDFs, we may conclude that the local bonding and chemical structures are similar for TiC-CDC-800-VAC and TiC-CDC-600-VAC. The differences in the $\Delta \delta$ values measured by NMR then must arise from differences in the carbon porosity, with the larger number of pores bigger than $1 \mathrm{~nm}$ diameter in TiC-CDC-800-VAC giving rise to a $\Delta \delta$ value of smaller magnitude. Such pore size effects have previously been predicted theoretically, ${ }^{18,20,23,24}$ and have also been observed experimentally. For example, Borchardt et al. observed pore size effects in experiments on mesoporous and microporous carbons, ${ }^{19}$ while Anderson et al. also observed a pore size effect in microporous carbons. ${ }^{18}$ Comparing TiC-CDC-400-VAC to the other annealed carbons, here the $\Delta \delta$ value has the largest magnitude of all. While this is consistent with the measured pore size distribution of this carbon, which shows it to have the smallest pores, it is unclear whether the structural differences revealed by the PDFs also have an effect on the $\Delta \delta$ value. 
a)<smiles>C1CCCOCC1</smiles>

$\mathrm{BF}_{4}-$ studied in-pore

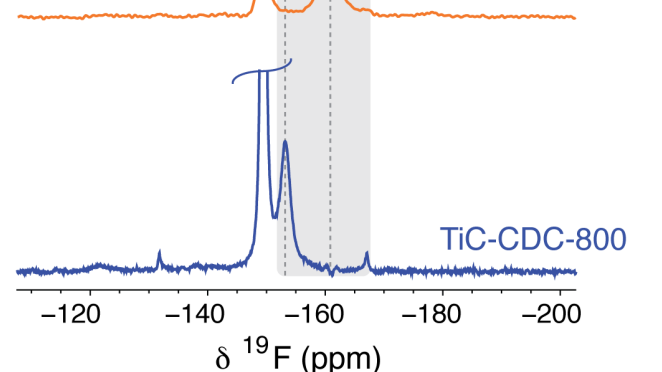

b)

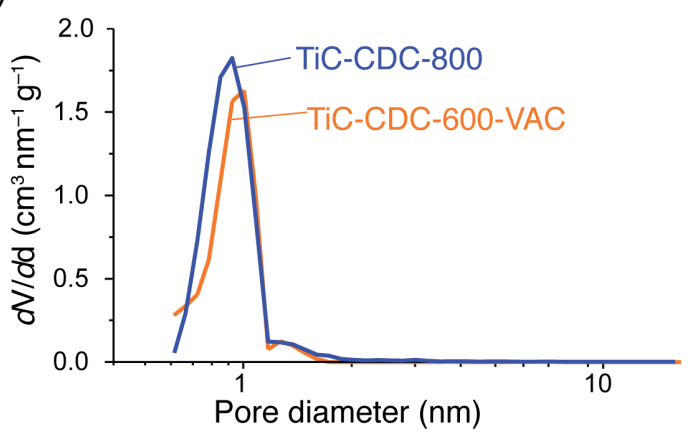

c)

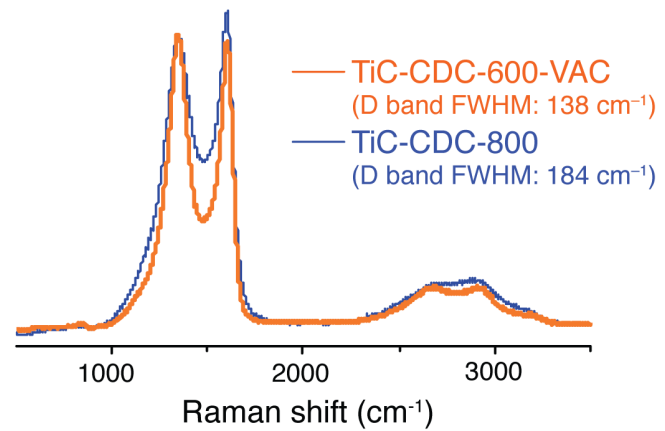

d)

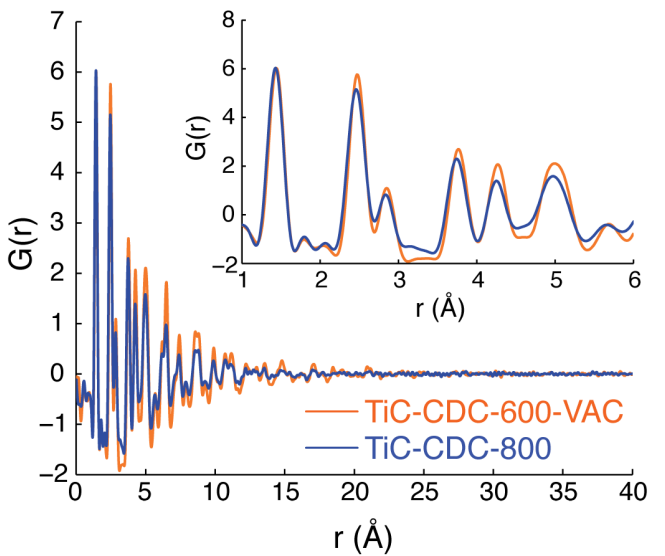

e)

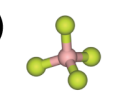
in-pore

$\mathrm{BF}_{4}^{-}$studied TiC-CDC-800-VAC

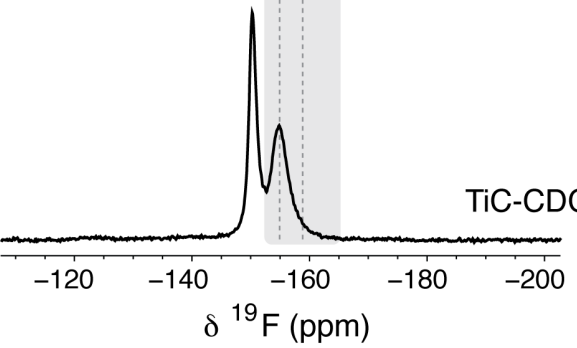

f)

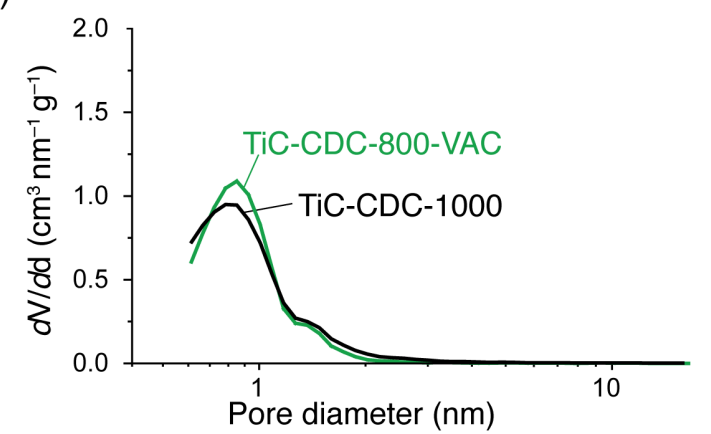

g)

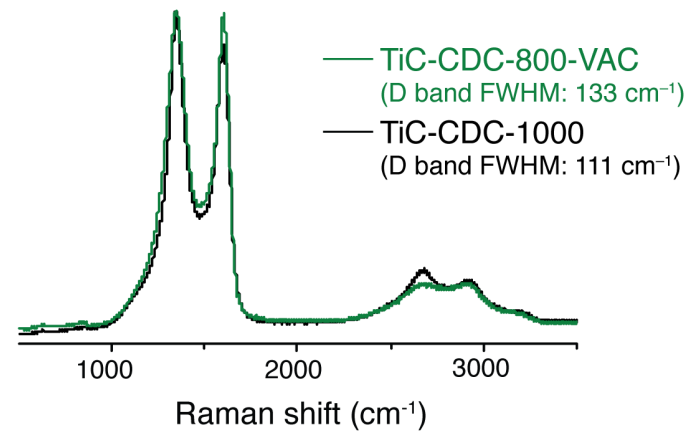

h)

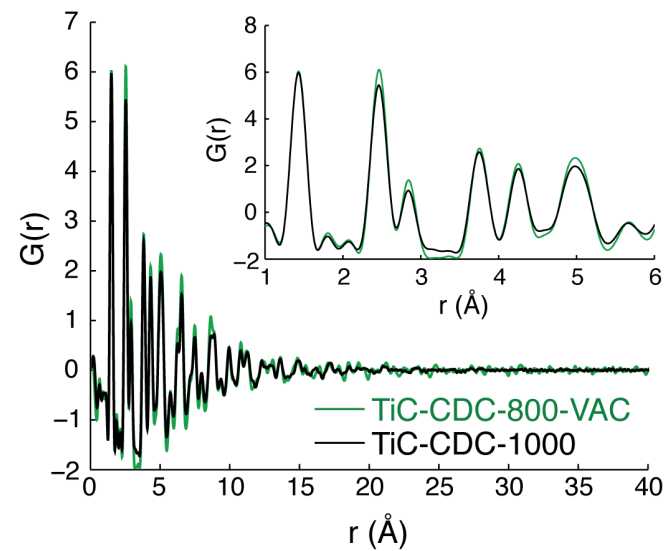

Figure 3 a) ${ }^{19} \mathrm{~F}$ MAS NMR $(7.1 \mathrm{~T})$ spectra of TiC-CDC-800 and TiC-CDC-600-VAC soaked with $\mathrm{NEt}_{4} \mathrm{BF}_{4} / \mathrm{dACN}^{(1.5}$ M) electrolyte. Dashed vertical lines show the positions of the in-pore resonances. b) Pore size distributions, c) Raman spectra and d) X-ray PDFs. In e) to h) analogous data is shown for TiC-CDC-1000 and TiC-CDC-800-VAC. Structural ordering in the carbon has a dramatic effect on the chemical shifts observed for in-pore ions.

To further investigate the effects of vacuum annealing on the carbon structures in the absence of pore size effects, we compared experimental data for TiC-CDC and TiC-CDC-VAC samples with similar 
pore size distributions (Figure 3). For TiC-CDC-800 and TiC-CDC-600-VAC, the NMR spectra are remarkably different despite the similar pore size distributions of the two carbons (Figure 3a,b). The $\Delta \delta$ value is significantly larger for the vacuum-annealed carbon than the non-annealed carbon, with respective values of -11.1 and $-3.9 \mathrm{ppm}$. Given the similar pore size distributions of these carbons, the increases of $\Delta \delta$ here must arise from increases in the sizes of carbon domains in which ring currents are present. This may originate from a combination of the formation of larger hexagonally bonded carbon domains, the annealing of defects and the removal of functional groups. The Raman spectra of these carbons also show significant differences and indicate that the carbon-carbon bonding in TiC-CDC-600-VAC is more uniform than in TiC-CDC-800, with the D-band FWHM smaller for the former (Figure 3c). The X-ray PDFs also reveal differences, with the PDF of the TiCCDC-600-VAC sample showing C-C correlations to slightly longer distances $(\sim 20-25 \AA)$ than the PDF of the TiC-CDC-800 sample ( 15-20 $)$. Vacuum annealing also appears to have removed some of the 7-membered rings from the structure, with a clear loss of intensity of the small correlation at $\sim 3.2 \AA$ observed for the annealed sample. The findings from the PDFs are consistent with the observations from NMR, the results together pointing towards the presence of larger hexagonally bonded carbon domains in the vacuum annealed carbon, while the Raman spectroscopy data also points toward more uniform carbon-carbon bonding in this material.

NMR experiments for a second pair of carbons (Figure 3e), again with similar pore size distributions, (Figure $3 \mathrm{f}$ ) also show a larger $\Delta \delta$ value for the annealed sample $(-9.7 \mathrm{ppm})$ than the non-annealed sample $(-5.5 \mathrm{ppm})$. Again, this difference arises from an increase in the size of the carbon domains in which ring currents are set up in the applied magnetic field. The difference in $\Delta \delta$ values for these two samples is smaller than the corresponding difference for TiC-CDC-800 and TiC-CDC-600-VAC, suggesting that the differences between the two samples are smaller in this case. The differences in the Raman spectra and X-ray PDFs are also much less pronounced for these two samples, with the Raman spectra nearly identical, with a slightly broader D-band for TiC-CDC-1000. These findings (Raman and PDF) suggest that the connectivity and bonding of carbon atoms in these materials is on the whole very similar, yet there are differences in the local electronic structure, manifested in different ring current effects (NMR). This may arise from the presence of more functional groups in the TiC-CDC-1000 sample, which may disrupt the conjugated $\pi$ system of the carbon. ${ }^{49}$ Recently, Xu et al. conducted a ${ }^{1} \mathrm{H}$ NMR study of the adsorption of water in two mesoporous carbons with similar pore size distributions. ${ }^{22}$ Their observation of larger ring current shifts for the carbon prepared at higher temperature reflects the effects observed here, again indicating increases in the size of the carbon domains in which ring currents are present. It is apparent that the maximum heat treatment temperature of the carbon is a key parameter in determining different structural features in porous carbons. 


\section{Modelling the carbon domain sizes with NMR and lattice simulations}

a) Max. heat treatment temperature $\left({ }^{\circ} \mathrm{C}\right)$

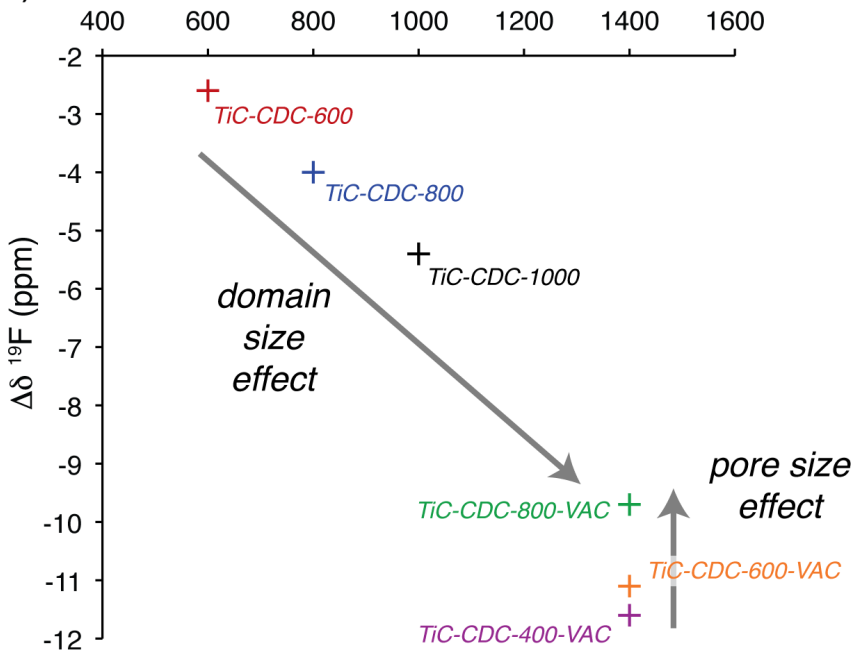

b)

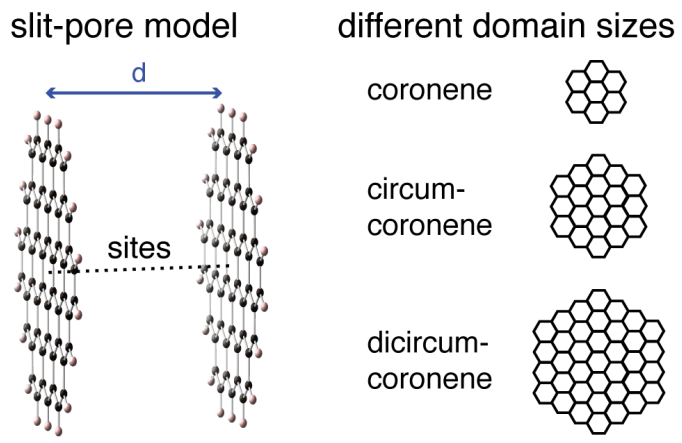

c)

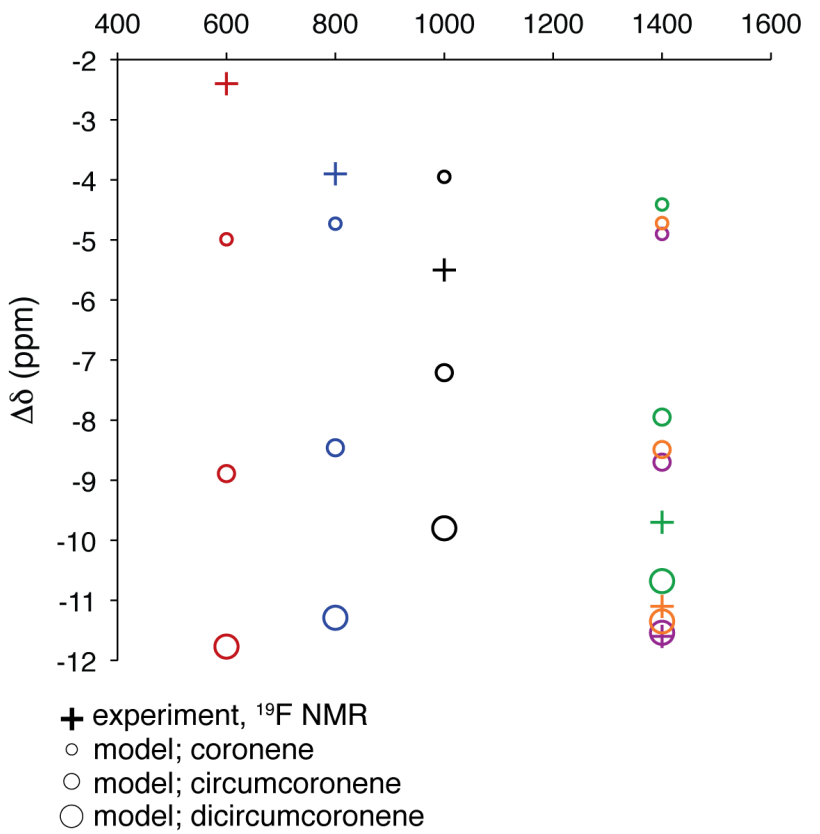

Figure 4 (a) Summary of $\otimes^{\mathrm{TM}}{ }^{19} \mathrm{~F}$ values measured in this work. (b) Schematic of structures used in lattice model simulations of $\otimes^{\mathrm{TM}}$ values. (c) Simulated and experimental $\otimes^{\mathrm{TM}}$ values. For each carbon, three different simulated $\otimes^{\mathrm{TM}}$ values are shown, corresponding to values computed with models employing coronenes of different sizes. The colour coding of the data points in (c) for the different carbons is given in (a).
To explore the relationship between $\Delta \delta$ values and carbon structure further, values are plotted against the maximum heat treatment temperature used in the synthesis of each sample in Figure 4a. As this temperature is increased, there is a general increase in the magnitude of the $\Delta \delta$ value, reflecting increases in the sizes of the carbon domains in which ring currents are established. For the vacuum annealed carbons $\left(1400{ }^{\circ} \mathrm{C}\right)$, the effects of porosity appear dominant, though this is a minor effect compared to increased carbon ordering for the range of carbons studied here.

The evolution of the carbon domain size with maximum heat treatment temperature was then investigated by simulating $\Delta \delta$ values for model carbon structures. In our lattice-type model, ${ }^{24}$ slit-pores are constructed from discrete $\mathrm{sp}^{2}$ hybridised carbon fragments from the coronene family (Figure 4b). This structural model has the benefits of being computationally easy to handle, while satisfying the key requirement of consisting of $\mathrm{sp}^{2}$-hybridised carbon with a nanoporous structure. In the model, a distribution of slitpores with different pore sizes are studied, such that the modelled pore size distribution exactly matches that obtained from the experimental $\mathrm{N}_{2}$ sorption isotherm. The inpore adsorption energies are defined using previous molecular dynamics simulation results, ${ }^{50}$ and the variation of the chemical shift with pore size follows from calculated NICS 
values $^{20}$ (see Supporting Information for more details).

The $\Delta \delta$ values were simulated for each carbon material (with its measured pore size distribution), with three separate simulations carried out for three coronenes with different sizes (Figure 4b). These different coronenes represent different characteristic domain sizes in the carbon (Figure 4c). We then compare the experimental $\Delta \delta$ values to the different simulated values to assess the sizes of the ring current domains in each carbon (with the pore size effects already accounted for in the simulations). According to our model, the effective average domain size of TiC-CDC-600 is somewhat less than that of coronene (7.5 $\AA$ diameter). For TiC-CDC-800, the domain size is similar to that of coronene, while for TiC-CDC-1000 the domain size is intermediate between that of coronene ( $7.5 \AA$ diameter) and circumcoronene (12.4 $\AA$ diameter). Following vacuum annealing, the $\Delta \delta$ values are best modelled by the dicircumcoronene (17.3 $\AA$ diameter) slit-pores (Figure 4c), with all carbons having a very similar characteristic coherence length. For the dicircumcoronene model, the simulated $\Delta \delta$ values are $-11.5,-11.4$ and $-10.7 \mathrm{ppm}$, for TiC-CDC-400-VAC, -600-VAC and -800-VAC, respectively, comparing reasonably well to the experimental values of $-11.6,-11.1$ and $-9.7 \mathrm{ppm}$. This supports the idea that the variations of the $\Delta \delta$ values for these vacuum-annealed carbons are dominated by the differences in the pore size distributions. Of note, the magnitude of the $\Delta \delta$ value is smaller for TiC-CDC-800-VAC than TiC-CDC-600-VAC in both the experiments and the simulations, despite TiC-CDC-800-VAC exhibiting its maximum in the pore distribution at $0.9 \mathrm{~nm}$, compared to $1.0 \mathrm{~nm}$ for TiC-CDC-600-VAC (Figure 2b). This is because TiC-CDC-800-VAC has a significant number of pores with sizes between 1.0 and $2.0 \mathrm{~nm}$ (Figure 2b), which significantly reduce the magnitude of the $\Delta \delta$ value due to the strong pore-size dependence of the ring current shifts. $^{20}$

The estimated ring current domain sizes here are similar to the length scales over which the carboncarbon coherences decay in the X-ray PDFs (typically between 15-25 $\AA$ ), though the variations of the ring current domains extracted from the NMR measurements seem more marked. This suggests that the variations of the local electronic structure of the carbon with maximum heat treatment temperature are more significant than the structural changes detected by the PDF. This may be due to variations in the numbers of defects, such as vacancies or functional groups, that will have a large effect on the electronic structure of the carbon, disrupting the carbon ring currents.

We note that the results presented in Figure 4c correspond to simulations using the isotropic chemical shift values calculated along a line perpendicular to the carbon planes and passing through the centre of the coronenes (Figure 4b), and the adsorption profiles of $\mathrm{BF}_{4}^{-}$anions in an organic electrolyte $\left(\mathrm{BMIBF}_{4}\right.$ in $\left.\mathrm{ACN}, 1.5 \mathrm{M}\right)$. The lateral dependence of the NICS and the influence of the adsorption 
profiles were investigated and were shown to lead to a small overall shift of all the $\Delta \delta$ values without changing the overall trends (see Supporting Information).

\section{Characterising activated carbons}

a)

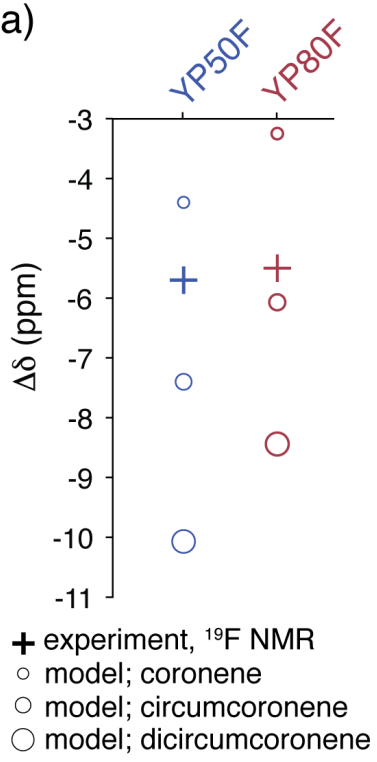

b)

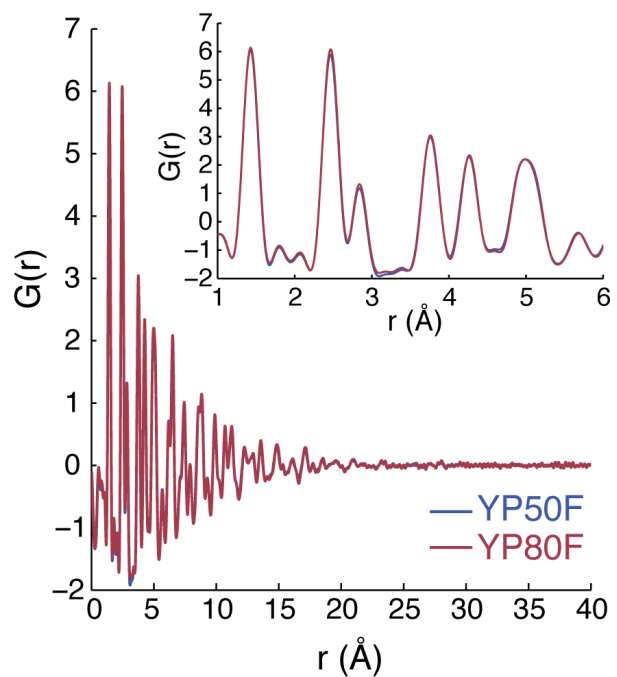

c)
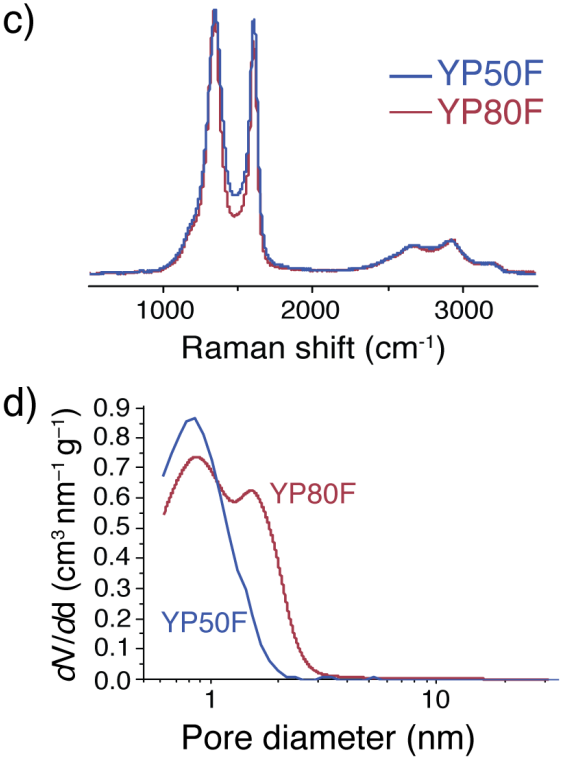

Figure 5 (a) Simulated and experimental $\Delta \delta$ values for YP50F and YP80F activated carbons. For each carbon, three different simulated $\Delta \delta$ values are shown, corresponding to values computed with models employing coronenes of different sizes. (b) X-ray PDFs, (c) Raman spectra and (d) pore size distributions of the carbons.

Our NMR approach can be used to characterise the structures of other porous carbon materials beyond CDCs. As was done above for the CDC materials, first the $\Delta \delta$ value and the carbon pore size distributions were measured experimentally, and then $\Delta \delta$ values were simulated for different carbon domain sizes. Experimental and simulated $\Delta \delta$ values for two commercial coconut shell-derived activated carbons, YP50F and YP80F (Kuraray, Japan) are shown in Figure 5a (NMR spectra are shown in the Supporting Information). Comparison of the experimental and simulated $\Delta \delta$ values indicates that the sizes of the carbon domains is similar for these carbons, with sizes intermediate between that of coronene $(12.4 \AA)$ and circumcoronene $(17.8 \AA)$, as with TiC-CDC-1000. For YP80F, the experimental data point is closer to the value simulated in the circumcoronene model than for YP50F, suggesting that YP80F has slightly larger domains. PDFs of these carbons (Figure $5 b)$ are almost identical, further supporting the idea that the different carbon sheets (pore walls) are not strongly correlated with each other, while the Raman spectra (Figure 5c) show some small differences, indicating that the carbon-carbon bonding is slightly more uniform in YP80F. It is interesting that the local chemical and electronic structures of these carbons are similar despite significant differences in the pore size distributions (Figure 5d). 
Overall, our findings in Figure 5 highlight how the NMR approach offers a new way to probe structural ordering in porous carbons, and can provide information that is complementary to Raman and PDF studies. Our slit-pore based model structures with discrete carbon fragments are a first step towards modelling the ring current shifts in realistic carbon structures. More accurate structural models will be used in future studies, to take into account the connectivity of carbon fragments and curvature in the sheets. Moreover, the small differences in the measured $\Delta \delta$ values for nuclei in different ions will be investigated such that accurate structural information can be extracted from the NMR spectra.

\section{Conclusions}

NMR spectroscopy and PDF analysis techniques offer new insights into the structures of porous carbon materials, complementing the more conventional Raman spectroscopy method. PDF analysis is a powerful probe of the local carbon structure, and reveals a prevalence of $\mathrm{sp}^{2}$-hybridised hexagonal carbon rings, as well as the presence of seven-membered carbon rings for TiC-CDCs prepared at low temperatures. Disorder in the carbon-carbon bonds can also be probed by the FWHMs of the correlations, showing good agreement with the interpretations of the Raman spectra.

In the NMR experiments, the differences between the in-pore and neat electrolyte chemical shifts are sensitive to the carbon pore size distribution (larger pores, smaller shift differences) and the sizes of the carbon domains in which the ring currents act (larger domains, larger shift differences), with the observed shift dominated by the domain size effect for the series of carbons studied here. By carrying out lattice simulations, the effects of the different pore size distributions can be accounted for, and the sizes of the ring current domains can be estimated. This approach represents a promising new way to characterize the local structure of porous carbon materials.

As the chlorine treatment temperature is raised from 600 to $1000{ }^{\circ} \mathrm{C}$ for TiC-CDCs, the carboncarbon bonding becomes more ordered, with less curved structures, and larger carbon domains in which ring currents act (on application of a magnetic field). Here, the NMR approach suggests that the sizes of the carbon domains increase significantly as the chlorine-treatment temperature is increased, while the correlations in the PDF show a less significant variation, with the correlations extending to $\sim 20 \AA$ in each case. Vacuum annealing at $1400{ }^{\circ} \mathrm{C}$ increases the uniformity of the local chemical bonding of the carbons, while further increasing the size of the carbon domains in which ring currents act. However, depending on the original chlorine treatment temperature, subtle differences in the carbon structures remain, hypothesised to arise from curvature effects. Overall, our study begins to disentangle the complexity of the structures of porous carbons and offers new approaches to probe local order. 


\section{Experimental details}

\section{Carbon synthesis}

Full details of the syntheses of TiC-CDC powder can be found elsewhere. ${ }^{33}$ Briefly, TiC was heated at the desired temperature $\left(600,800\right.$, or $\left.1000^{\circ} \mathrm{C}\right)$ in dry chlorine gas for $3 \mathrm{~h}$. The sample was then held at $600^{\circ} \mathrm{C}$ in hydrogen gas for $2 \mathrm{~h}$ to remove chlorine and chloride residues. Samples treated in chlorine gas at a temperature $\mathrm{X}^{\circ} \mathrm{C}\left(e . g ., 600{ }^{\circ} \mathrm{C}\right)$ are referred to as TiC-CDC-X (e.g., TiC-CDC-600).

Vacuum treatment of the CDC samples was carried out in a water-cooled Thermal Technology (USA) furnace system (Model 1100-3580-W1) employing tungsten heaters and a pressure of $10^{-4}$ to $10^{-5}$ mbar. Sample material was placed in graphite ingots and the heating and cooling rates were 10 ${ }^{\circ} \mathrm{C} \cdot \mathrm{min}^{-1}$. The heating process first employed a holding period of $2 \mathrm{~h}$ at $200{ }^{\circ} \mathrm{C}$ to remove adsorbed water and a final high-temperature annealing step at $1400{ }^{\circ} \mathrm{C}$ for $1 \mathrm{~h}$.

YP50F and YP80F activated carbon powders were obtained from Kuraray (Japan) and were used without further treatment.

Free-standing carbon films were fabricated using the standard method for preparing film electrodes, by mixing carbon powder (95 wt.\%) and polytetrafluoroethylene (PTFE) binder (5 wt.\%) in ethanol, as described in more detail elsewhere. ${ }^{33}$

\section{NMR experiments}

Pieces of carbon film ( $\sim 6 \mathrm{mg})$ were cut out and dried for at least $15 \mathrm{~h}$ at $200{ }^{\circ} \mathrm{C}$ in vacuo before being transferred to an argon glove box. These were packed into $2.5 \mathrm{~mm}$ outer-diameter zirconia magic angle spinning (MAS) rotors, to which $\sim 4 \mu \mathrm{L}$ of tetraethylammonium tetrafluoroborate, $\mathrm{NEt}_{4} \mathrm{BF}_{4},(\geq$ $99.0 \%$, Sigma Aldrich) $1.5 \mathrm{M}$ in deuterated acetonitrile, $\mathrm{D}_{3} \mathrm{CCN},(99.80 \%$, Eurisotop, referred to as $\mathrm{dACN}$ here) was added using a micro-syringe.

NMR experiments were performed using a Bruker Avance spectrometer operating at a magnetic field strength of 7.1 T, corresponding to a ${ }^{1} \mathrm{H}$ Larmor frequency of $300.2 \mathrm{MHz}$. A Bruker $2.5 \mathrm{~mm}$ double resonance MAS probe was used. All NMR experiments used spin-echo sequences with $5 \mathrm{kHz}$ MAS and a single rotor period $(200 \mu \mathrm{s})$ was used for the echo delay. ${ }^{19} \mathrm{~F}$ NMR spectra were referenced relative to neat hexafluorobenzene $\left(\mathrm{C}_{6} \mathrm{~F}_{6}\right)$ at $-164.9 \mathrm{ppm}$, while ${ }^{1} \mathrm{H}$ NMR spectra were referenced relative to tetramethylsilane using the $\mathrm{CH}_{3}$ resonance of liquid ethanol at $1.2 \mathrm{ppm}$ as a secondary reference. Radiofrequency strengths of 100 and $109 \mathrm{kHz}$ were used for ${ }^{1} \mathrm{H}$ and ${ }^{19} \mathrm{~F}$ experiments, respectively, on vacuum annealed carbons, while powers of 98 and $82 \mathrm{kHz}$ were used on nonannealed samples. 


\section{Nitrogen sorption analysis}

Nitrogen sorption analysis was carried out using Quantachrome Instruments (USA) Autosorb system at $-196{ }^{\circ} \mathrm{C}$. Pore size distributions (PSDs) were determined using the quenched solid density functional theory (QSDFT) method ${ }^{37}$ on the desorption branch of the isotherms. All samples were predominantly microporous with a small volume fraction of the total pore volume associated with pores larger than $2 \mathrm{~nm}$. The cut-off of the nitrogen gas sorption analysis is around $30 \mathrm{~nm}$ so that no inter-particle pores between the CDC grains are considered. Nitrogen sorption isotherms are shown in the Supporting Information.

\section{Raman spectroscopy}

Raman spectra were measured using a Renishaw inVia Raman system equipped with an Nd-YAG laser and an excitation wavelength of $532 \mathrm{~nm}$ with power of $0.2 \mathrm{~mW}$ at the focal point focused on a spot of ca. $2 \mu \mathrm{m}$ diameter. A grating with 2400 lines $/ \mathrm{mm}$ and a 50x objective (numeric aperture: 0.9) were used, yielding a spectral resolution of $1.2 \mathrm{~cm}^{-1}$. Raman peak fitting was accomplished assuming two Lorentzian peaks, one for the D-mode and one for the G-mode, respectively (see Supporting Information for full results).

\section{Pair distribution function analysis}

Total-scattering data were collected at beamline 11-ID-B at the Advanced Photon Source, Argonne National Laboratory using an X-ray energy of $86 \mathrm{keV}$ and a large area amorphous silicon detector (Perkin-Elmer). Samples were packed in 0.0395 inch inner diameter Kapton capillaries (ColeParmer). Scattering images were reduced to one-dimensional data using FIT2D, ${ }^{51}$ using $\mathrm{CeO}_{2}$ as a calibration standard. The data were corrected for background scattering, Compton scattering and detector effects, and Fourier Transformed (using a $\mathrm{Q}_{\max }$ of $24 \AA^{-1}$ ) using PDFGetX2. ${ }^{52}$ Simulations of the PDF were performed using PDFGui. Peak fitting was performed in Fityk, ${ }^{53}$ using Gaussian peaks.

\section{Lattice simulations of NMR chemical shifts}

Full details of the lattice simulations used to simulate $\Delta \delta$ values are given in the Supporting Information.

\section{Supporting Information Available:}

Gas sorption isotherms, ${ }^{1} \mathrm{H}$ NMR spectra, FWHMs from PDFs, PDF simulation, evidence for 7membered carbon rings, fitted Raman parameters, X-ray diffraction patterns, details of lattice 
simulations, and NMR spectra for YP50F and YP80F. This material is available free of charge via the Internet at http://pubs.acs.org

\section{Acknowledgements:}

A.C.F., J.M.G., C.M., P.K.A, E.K.H. and C.P.G. acknowledge the Sims Scholarship (A.C.F.), EPSRC (via the Supergen consortium, J.M.G.), and the EU ERC (via an Advanced Fellowship to C.P.G.) for funding. C.M. and P.K.A. acknowledges the School of the Physical Sciences of the University of Cambridge for funding through an Oppenheimer Research Fellowship. P.K.A acknowledges a Junior Research Fellowship from Gonville and Caius College, Cambridge. A.C.F. and J.M.G. thank the NanoDTC Cambridge for travel funding. M.A., M.Z., and V.P. acknowledge funding from the German Federal Ministry for Research and Education (BMBF) in support of the nanoEES ${ }^{3 \mathrm{D}}$ project (award number 03EK3013) as part of the strategic funding initiative energy storage framework and kindly thank Prof. Arzt (INM) for his continuing support. CDC work at Drexel University (Y.G.) was supported by the Fluid Interface Reactions, Structures and Transport (FIRST) Center, an Energy Frontier Research Center funded by the U.S. Department of Energy, Office of Science, Office of Basic Energy Sciences. This research used resources of the Advanced Photon Source, a U.S. Department of Energy (DOE) Office of Science User Facility operated for the DOE Office of Science by Argonne National Laboratory under Contract No. DE-AC02-06CH11357. We thank Daan Frenkel for his contributions to this work, and Boris Dyatkin for comments on the manuscript.

\section{References:}

(1) Marsh, H.; Rodriguez-Reinoso, F. Activated Carbon; 1st ed.; Elsevier Sci. Ltd., 2006.

(2) Simon, P.; Gogotsi, Y. Charge Storage Mechanism in Nanoporous Carbons and Its Consequence for Electrical Double Layer Capacitors. Phil. Trans. R. Soc. A 2010, 368, 34573467.

(3) Porada, S.; Zhao, R.; van der Wal, A.; Presser, V.; Biesheuvel, P. M. Review on the Science and Technology of Water Desalination by Capacitive Deionization. Prog. Mater. Sci. 2013, $58,1388-1442$.

(4) Harris, P. New Perspectives on the Structure of Graphitic Carbons. Crit. Rev. Solid State Mater. Sci. 2005, 30, 235-253.

(5) Harris, P. J. F. Fullerene-like Models for Microporous Carbon. J. Mater. Sci. 2013, 48, 565577.

(6) Franklin, R. E. The Structure of Graphitic Carbons. Acta Cryst. 1951, 4, 253-261.

(7) Franklin, R. E. Crystallite Growth in Graphitizing and Non-Graphitizing Carbons. Proc. R. Soc. A 1951, 209, 196-218.

(8) Billinge, S.; Thorpe, M. Local Structure from Diffraction; Plenum New York, 1998.

(9) Kane, M. S.; Goellner, J. F.; Foley, H. C.; Difrancesco, R.; Billinge, S. J. L.; Lansing, E.; Allard, L. F. Symmetry Breaking in Nanostructure Development of Carbogenic Molecular 
Sieves : Effects of Morphological Pattern Formation on Oxygen and Nitrogen Transport. Chem. Mater. 1996, 8, 2159-2171.

(10) Petkov, V.; Difrancesco, R. G.; Billinge, S. J. L.; Acharya, M.; Foley, H. C. Local Structure of Nanoporous Carbons. Phil. Mag. B 1999, 79, 1519-1530.

(11) Acharya, M.; Strano, M. S.; Mathews, J. P.; Billinge, S. J. L.; Petkov, V.; Subramoney, S.; Foley, H. C. Simulation of Nanoporous Carbons: A Chemically Constrained Structure. Phil. Mag. B 1999, 79, 1499-1518.

(12) Dash, R.; Chmiola, J.; Yushin, G.; Gogotsi, Y.; Laudisio, G.; Singer, J.; Fischer, J.; Kucheyev, S. Titanium Carbide Derived Nanoporous Carbon for Energy-Related Applications. Carbon N. Y. 2006, 44, 2489-2497.

(13) Harris, P. J. F.; Liu, Z.; Suenaga, K. Imaging the Atomic Structure of Activated Carbon. J. Phys. Condens. Matter 2008, 20,362201.

(14) Osswald, S.; Chmiola, J.; Gogotsi, Y. Structural Evolution of Carbide-Derived Carbons upon Vacuum Annealing. Carbon N. Y. 2012, 50, 4880-4886.

(15) Kumar, A.; Lobo, R.; Wagner, N. Porous Amorphous Carbon Models from Periodic Gaussian Chains of Amorphous Polymers. Carbon N. Y. 2005, 43, 3099-3111.

(16) Jain, S. K.; Pellenq, R. J.; Pikunic, J. P.; Gubbins, K. E. Molecular Modeling of Porous Carbons Using the Hybrid Reverse Monte Carlo Method. Langmuir 2006, 24, 9942-9948.

(17) Palmer, J.; Jain, S.; Gubbins, K. E.; Cohaut, N.; Fischer, J. E.; Dash, R. K.; Gogotsi, Y. Hybrid Reverse Monte Carlo Simulations of Microporous Carbons. Charact. Porous Solids VIII, Proc. 8th Int. Conf. Charact. Porous Solids, Eds. S. Kaskel, P. Llewellyn, F. Rodriguez-Reinoso N.A. Seaton, pp. 56-63, R. Soc. Chem. Cambridge, Spec. Pub 2009, 318, 56-63.

(18) Anderson, R. J.; McNicholas, T. P.; Kleinhammes, A.; Wang, A.; Liu, J.; Wu, Y. NMR Methods for Characterizing the Pore Structures and Hydrogen Storage Properties of Microporous Carbons. J. Am. Chem. Soc. 2010, 132, 8618-8626.

(19) Borchardt, L.; Oschatz, M.; Paasch, S.; Kaskel, S.; Brunner, E. Interaction of Electrolyte Molecules with Carbon Materials of Well-Defined Porosity: Characterization by Solid-State NMR Spectroscopy. Phys. Chem. Chem. Phys. 2013, 15, 15177-15184.

(20) Forse, A. C.; Griffin, J. M.; Presser, V.; Gogotsi, Y.; Grey, C. P. Ring Current Effects: Factors Affecting the NMR Chemical Shift of Molecules Adsorbed on Porous Carbons. J. Phys. Chem. C 2014, 118, 7508-7514.

(21) Wang, H.; Kleinhammes, A.; Mcnicholas, T. P.; Liu, J.; Wu, Y. Water Adsorption in Nanoporous Carbon Characterized by in Situ NMR : Measurements of Pore Size and Pore Size Distribution. J. Phys. Chem. C 2014, 118, 8474-8480.

(22) Xu, Y.; Watermann, T.; Limbach, H.-H.; Gutmann, T.; Sebastiani, D.; Buntkowsky, G. Water and Small Organic Molecules as Probes for Geometric Confinement in Well-Ordered Mesoporous Carbon Materials. Phys. Chem. Chem. Phys. 2014, 16, 9327-9336.

(23) Xing, Y.-Z.; Luo, Z.-X.; Kleinhammes, A.; Wu, Y. Probing Carbon Micropore Size Distribution by Nucleus Independent Chemical Shift. Carbon N. Y. 2014, 77, 1132-1139.

(24) Merlet, C.; Forse, A. C.; Griffin, J. M.; Frenkel, D.; Grey, C. P. Lattice Simulation Method to Model Diffusion and NMR Spectra in Porous Materials. J. Chem. Phys. 2015, 142, 094701.

(25) McNicholas, T. P.; Wang, A.; Neill, K. O.; Anderson, R. J.; Stadie, N. P.; Kleinhammes, A.; Parilla, P.; Simpson, L.; Ahn, C. C.; Wang, Y.; et al. H2 Storage in Microporous Carbons from PEEK Precursors. J. Phys. Chem. C 2010, 114, 13902-13908.

(26) Liu, X.; Pan, X.; Shen, W.; Ren, P.; Han, X.; Bao, X. NMR Study of Preferential Endohedral Adsorption of Methanol in Multiwalled Carbon Nanotubes. J. Phys. Chem. C 2012, 116, 7803-7809.

(27) Wang, H.; Köster, T. K.-J.; Trease, N. M.; Ségalini, J.; Taberna, P.-L.; Simon, P.; Gogotsi, Y.; Grey, C. P. Real-Time NMR Studies of Electrochemical Double-Layer Capacitors. J. Am. Chem. Soc. 2011, 133, 19270-19273.

(28) Wang, H.; Forse, A. C.; Griffin, J. M.; Trease, N. M.; Trognko, L.; Taberna, P.-L.; Simon, P.; Grey, C. P. In Situ NMR Spectroscopy of Supercapacitors: Insight into the Charge Storage Mechanism. J. Am. Chem. Soc. 2013, 135, 18968-18980. 
(29) Griffin, J. M.; Forse, A. C.; Wang, H.; Trease, N. M.; Simon, P.; Grey, C. P. Ion Counting in Supercapacitor Electrodes Using NMR Spectroscopy. Faraday Disc. 2014, 176, 49-68.

(30) Luo, Z.-X.; Xing, Y.-Z.; Ling, Y.-C.; Kleinhammes, A.; Wu, Y. Electroneutrality Breakdown and Specific Ion Effects in Nanoconfined Aqueous Electrolytes Observed by NMR. Nat. Comm. 2015, 6, DOI:10.1038/ncomms7358.

(31) Griffin, J. M.; Forse, A. C.; Tsai, W .-Y.; Taberna, P.-L.; Simon, P.; Grey, C. P. In Situ NMR and Electrochemical Quartz Crystal Microbalance Reveal the Structure of the Electric DoubleLayer in Supercapacitor Electrodes. Nat. Mater. 2015, 14, 812-819.

(32) Harris, R. K.; Thompson, T. V.; Norman, P. R.; Pottage, C. Phosphorus-31 NMR Studies of Adsorption onto Activated Carbon. Carbon N. Y. 1999, 37, 1425-1430.

(33) Forse, A. C.; Griffin, J. M.; Wang, H.; Trease, N. M.; Presser, V.; Gogotsi, Y.; Simon, P.; Grey, C. P. Nuclear Magnetic Resonance Study of Ion Adsorption on Microporous CarbideDerived Carbon. Phys. Chem. Chem. Phys. 2013, 15, 7722-7730.

(34) Ilott, A.; Trease, N.; Grey, C.; Jerschow, A. Multinuclear in Situ Magnetic Resonance Imaging of Electrochemical Double-Layer Capacitors. Nat. Comm. 2014, 5, 4536.

(35) Sebastiani, D. Current Densities and Nucleus-Independent Chemical Shift Maps from Reciprocal-Space Density Functional Perturbation Theory Calculations. Chem. Phys. Chem. 2006, 7, 164-175.

(36) Kibalchenko, M.; Payne, M. C.; Yates, J. R. Magnetic Response of Single-Walled Carbon Nanotubes Induced by an External Magnetic Field. ACS Nano 2011, 5, 537-545.

(37) Neimark, A. V.; Lin, Y.; Ravikovitch, P. I.; Thommes, M. Quenched Solid Density Functional Theory and Pore Size Analysis of Micro-Mesoporous Carbons. Carbon N. Y. 2009, 47, 16171628.

(38) Lesplade, P.; Al-Jishi, R.; Dresselhaus, M. S. Model for Raman Scattering from Incompletely Graphitized Carbons. Carbon N. Y. 1982, 20, 427-431.

(39) Pimenta, M. A.; Dresselhaus, G.; Dresselhaus, M. S.; Cançado, L. G.; Jorio, A.; Saito, R. Studying Disorder in Graphite-Based Systems by Raman Spectroscopy. Phys. Chem. Chem. Phys. 2007, 9, 1276-1291.

(40) Ferrari, A. C.; Robertson, J. Interpretation of Raman Spectra of Disordered and Amorphous Carbon. Phys. Rev. B 2000, 61, 14095-14107.

(41) Zickler, G. A.; Smarsly, B.; Gierlinger, N.; Peterlik, H.; Paris, O. A Reconsideration of the Relationship between the Crystallite Size La of Carbons Determined by X-Ray Diffraction and Raman Spectroscopy. Carbon N. Y. 2006, 44, 3239-3246.

(42) Tuinstra, F.; Koenig, J. L. Raman Spectrum of Graphite. J. Chem. Phys. 1970, 53, 1126-1130.

(43) Urbonaite, S.; Hälldahl, L.; Svensson, G. Raman Spectroscopy Studies of Carbide Derived Carbons. Carbon N. Y. 2008, 46, 1942-1947.

(44) Dyatkin, B.; Gogotsi, Y. Effects of Structural Disorder and Surface Chemistry on Electric Conductivity and Capacitance of Porous Carbon Electrodes. Faraday Discuss. 2014, 172, 139-162.

(45) Forse, A. C.; Griffin, J. M.; Merlet, C.; Bayley, P. M.; Wang, H.; Simon, P.; Grey, C. P. NMR Study of Ion Dynamics and Charge Storage in Ionic Liquid Supercapacitors. J. Am. Chem. Soc. 2015, 137, 7231-7242.

(46) Vora, P.; Gopu, P.; Rosario-Canales, M.; Pérez, C.; Gogotsi, Y.; Santiago-Avilés, J.; Kikkawa, J. Correlating Magnetotransport and Diamagnetism of sp2-Bonded Carbon Networks through the Metal-Insulator Transition. Phys. Rev. B 2011, 84, 155114-155118.

(47) Palmer, J. C.; Llobet, A.; Yeon, S.-H.; Fischer, J. E.; Shi, Y.; Gogotsi, Y.; Gubbins, K. E. Modeling the Structural Evolution of Carbide-Derived Carbons Using Quenched Molecular Dynamics. Carbon N. Y. 2010, 48, 1116-1123.

(48) Chmiola, J.; Yushin, G.; Gogotsi, Y.; Portet, C.; Simon, P.; Taberna, P.-L. Anomalous Increase in Carbon Capacitance at Pore Sizes Less than 1 Nanometer. Science (80-. ). 2006, 313, 1760-1763.

(49) Takashiro, J.; Kudo, Y.; Kaneko, S.; Takai, K.; Ishii, T.; Kyotani, T.; Enoki, T.; Kiguchi, M. Heat Treatment Effect on the Electronic and Magnetic Structures of Nanographene Sheets 
Investigated through Electron Spectroscopy and Conductance Measurements. Phys. Chem. Chem. Phys. 2014, 16, 7280-7289.

(50) Merlet, C.; Salanne, M.; Rotenberg, B.; Madden, P. A. Influence of Solvation on the Structural and Capacitive Properties of Electrical Double Layer Capacitors. Electrochim. Acta 2013, 101, 262-271.

(51) Hammersley, A. P.; Svensson, S. O.; Hanfland, M.; Fitch, A. N.; Häusermann, D. TwoDimensional Detector Software: From Real Detector to Idealised Image or Two-Theta Scan. High Press. Res. 1996, 14, 235-248.

(52) Farrow, C. L.; Juhas, P.; Liu, J. W.; Bryndin, D.; Božin, E. S.; Bloch, J.; Proffen, T.; Billinge, S. J. L. PDFfit2 and PDFgui: Computer Programs for Studying Nanostructure in Crystals. J. Phys. Condens. Matter 2007, 19, 335219.

(53) Wojdyr, M. Fityk: A General-Purpose Peak Fitting Program. J. Appl. Cryst. 2010, 43, 11261128. 